Bull. Korean Math. Soc. 50 (2013), No. 2, pp. 459-467

http://dx.doi.org/10.4134/BKMS.2013.50.2.459

\title{
MATLIS INJECTIVE MODULES
}

\author{
HANGYU YAN
}

\begin{abstract}
In this paper, Matlis injective modules are introduced and studied. It is shown that every $R$-module has a (special) Matlis injective preenvelope over any ring $R$ and every right $R$-module has a Matlis injective envelope when $R$ is a right Noetherian ring. Moreover, it is shown that every right $R$-module has an $\mathcal{F}^{\perp_{1}}$-envelope when $R$ is a right Noetherian ring and $\mathcal{F}$ is a class of injective right $R$-modules.
\end{abstract}

\section{Introduction}

Throughout this paper, $R$ will denote an associative ring with identity and all modules will be unitary right $R$-modules.

The motivation of this paper is from [4], where the notion of Whitehead modules was studied. Recall that an $R$-module $M$ is called a Whitehead module or $W$-module if $\operatorname{Ext}_{R}^{1}(M, R)=0$. We introduce the notion of Matlis injective modules as a dual notion of Whitehead modules in some sense. An $R$-module $M$ is called Matlis injective if $\operatorname{Ext}_{R}^{1}(E(R), M)=0$, where $E(R)$ denotes the injective envelope of $R$. Let $R$ be an integral domain and $Q$ its field of quotients, an $R$-module $C$ is called Matlis cotorsion or weakly cotorson if $\operatorname{Ext}_{R}^{1}(Q, C)=0$. Then, it is easy to see that the notion of Matlis injective $R$-modules coincides with the notion of Matlis cotorsion $R$-modules when $R$ is an integral domain. Following [7], an $R$-module $M$ is called copure injective if $\operatorname{Ext}_{R}^{1}(E, M)=0$ for any injective $R$-module $E$. Clearly, every copure injective $R$-module is Matlis injective, but it is easy to see that the converse is not true in general. Thus Matlis injective $R$-modules can be seen as a generalization of copure injective $R$-modules.

Let $\mathcal{C}$ be a class of $R$-modules. Enochs defined a $\mathcal{C}$-(pre)cover $(\mathcal{C}$-(pre)envelope) of an $R$-module in [6]. Therefore, it is natural to study the existence of Matlis injective (pre)covers and Matlis injective (pre)envelopes. Obviously, the class of Matlis injective $R$-modules is closed under direct summands, but we show that it is not closed under direct sums in general. So there exist a ring

Received October 15, 2011; Revised January 20, 2012.

2010 Mathematics Subject Classification. 16D10, 16E30.

Key words and phrases. Matlis injective module, (pre)envelope, $\Sigma$-pure injective.

This research was partially supported by Research Fund of China Pharmaceutical University (No. 211148). 
$R$ and an $R$-module $M$ such that $M$ doesn't have a Matlis injective precover. Then, we are only interested in the existence of Matlis injective (pre)-envelopes in this paper. Let $\mathcal{F}$ be a class of $R$-modules, we denote by $\mathcal{F}^{\perp_{1}}$ the class of $R$-modules $N$ such that $\operatorname{Ext}_{R}^{1}(F, N)=0$ for every $F \in \mathcal{F}$. In [5, Theorem 10], Eklof and Trlifaj proved that if there is a set $\mathcal{S}$ of $R$-modules such that $\mathcal{F}^{\perp_{1}}=\mathcal{S}^{\perp_{1}}$, then every $R$-module has an $\mathcal{F}^{\perp_{1}}$-preenvelope. Using this result, we show that every $R$-module has a Matlis injective preenvelope. If $R$ is a right Noetherian ring, we show that every $R$-module has an $\mathcal{F}^{\perp_{1}}$-envelope, where $\mathcal{F}$ is any subclass of the class of injective $R$-modules. As a byproduct, we show that every $R$-module has a Matlis injective envelope when $R$ is a right Noetherian ring.

\section{Preliminaries}

In this section we briefly recall some definitions and results required in this paper.

For a ring $R, \operatorname{Mod}-R$ will denote the category of all right $R$-modules and $p d(M)$ will denote the projective dimension of $M$. For an $R$-module $M$, we denote by $E(M)$ the injective envelope of $M$. We frequently identify $M$ with its image in $E(M)$ and think of $M$ as a submodule of $E(M)$.

Let $\mathcal{C} \subseteq$ Mod- $R$. Define

$$
\begin{aligned}
& \mathcal{C}^{\perp_{1}}=\left\{X \in \operatorname{Mod}-R \mid \operatorname{Ext}_{R}^{1}(C, X)=0 \text { for all } C \in \mathcal{C}\right\}, \\
& { }^{\perp_{1}} \mathcal{C}=\left\{X \in \operatorname{Mod}-R \mid \operatorname{Ext}_{R}^{1}(X, C)=0 \text { for all } C \in \mathcal{C}\right\} .
\end{aligned}
$$

$\operatorname{Add}(\mathcal{C})=\left\{X \in \operatorname{Mod}-R \mid X\right.$ is a direct summand of $\bigoplus_{i \in I} C_{i}$, where $I$ is a set and where for any $i \in I, C_{i}$ is isomorphic to an element of $\left.\mathcal{C}\right\}$.

For $\mathcal{C}=\{C\}$, we write $C^{\perp_{1}},{ }^{\perp_{1}} C$ and $\operatorname{Add}(C)$ in place of $\{C\}^{\perp_{1}},{ }^{\perp_{1}}\{C\}$ and $\operatorname{Add}(\{C\})$, respectively.

Let $M \in \operatorname{Mod}-R$. A homomorphism $f \in \operatorname{Hom}_{R}(M, C)$ with $C \in \mathcal{C}$ is called a $\mathcal{C}$-preenvelope of $M$ provided that the abelian group homomorphism $\operatorname{Hom}_{R}\left(f, C^{\prime}\right): \operatorname{Hom}_{R}\left(C, C^{\prime}\right) \rightarrow \operatorname{Hom}_{R}\left(M, C^{\prime}\right)$ is surjective for each $C^{\prime} \in \mathcal{C}$. The $\mathcal{C}$-preenvelope $f$ is called a $\mathcal{C}$-envelope of $M$ provided that $f=g f$ implies $g$ is an automorphism for each $g \in \operatorname{End}_{R}(C)$. Moreover, a $\mathcal{C}$-preenvelope $f: M \rightarrow C$ of $M$ is called special provided that $f$ is injective and Coker $f \in{ }^{\perp_{1}} \mathcal{C}$. $\mathcal{C}$-envelopes may not exist in general, but if they exist, they are unique up to isomorphism. If $\mathcal{C}$ is the class of injective modules, then we get the usual injective envelopes.

$\mathcal{C}$-precovers and $\mathcal{C}$-covers are defined dually. These generalize the projective covers introduced by Bass in the 1960's.

A pair $(\mathcal{A}, \mathcal{B})$ of $R$-module classes is called a cotorsion theory (or cotorsion pair) provided that $\mathcal{A}^{\perp_{1}}=\mathcal{B}$ and $\mathcal{A}={ }^{\perp_{1}} \mathcal{B}$. An $R$-module $M$ is called cotorsion if $\operatorname{Ext}_{R}^{1}(F, M)=0$ for any flat $R$-module $F$. Let $\mathscr{F}$ be the class of flat $R$-modules and $\mathscr{C}$ be the class of cotorsion $R$-modules, it is known that $(\mathscr{F}, \mathscr{C})$ is a cotorsion theory.

For any class $\mathcal{F}$ of $R$-modules. The following theorem, due to Eklof and 
Trlifaj, says that every $R$-module has a special $\mathcal{F}^{\perp_{1}}$-preenvelope if there is a set $\mathcal{S}$ of $R$-modules such that $\mathcal{S}^{\perp_{1}}=\mathcal{F}^{\perp_{1}}$. Before stating the result, we need more notions:

A sequence of modules $\mathcal{A}=\left(A_{\alpha} \mid \alpha \leq \mu\right)$ is called a continuous chain of modules provided that $A_{0}=0, A_{\alpha} \subseteq A_{\alpha+1}$ for all $\alpha<\mu$ and $A_{\alpha}=\bigcup_{\beta<\alpha} A_{\beta}$ for all limit ordinals $\alpha \leq \mu$.

Let $M$ be a module and $\mathcal{C}$ a class of modules. Then $M$ is called $\mathcal{C}$-filtered provided that there are an ordinal $\kappa$ and a continuous chain, $\left(M_{\alpha} \mid \alpha \leq \kappa\right)$, consisting of submodules of $M$ such that $M=M_{\kappa}$, and such that each of the modules $M_{\alpha+1} / M_{\alpha}(\alpha<\kappa)$ is isomorphic to an element of $\mathcal{C}$. The chain $\left(M_{\alpha} \mid \alpha \leq \kappa\right)$ is called a $\mathcal{C}$-filtration of $M$.

Theorem 2.1 ([10], Theorem 3.2.1, p. 117). Let $\mathcal{S}$ be a set of $R$-modules and $M$ an $R$-module. Then there is a short exact sequence $0 \rightarrow M \hookrightarrow P \rightarrow N \rightarrow 0$, where $P \in \mathcal{S}^{\perp_{1}}$ and $N$ is $\mathcal{S}$-filtered. In particular, $M \hookrightarrow P$ is a special $\mathcal{S}^{\perp_{1}}$ preenvelope of $M$.

The following theorem from [10] gives a criterion to judge when an $R$-module $M$ has a $\mathcal{C}^{\perp_{1}}$-envelope.

Theorem 2.2 ([10], Theorem 2.3.2, p. 107). Let $R$ be a ring and $M$ be an $R$-module. Let $\mathcal{C}$ be a class of $R$-modules closed under extensions and direct limits. Assume that $M$ has a special $\mathcal{C}^{\perp_{1}}$-preenvelope $\nu$ with Coker $\nu \in \mathcal{C}$. Then $M$ has a $\mathcal{C}^{\perp_{1}}$-envelope.

A short exact sequence $0 \rightarrow A \rightarrow B \rightarrow C \rightarrow 0$ of $R$-modules is called pure if the induced sequence $0 \rightarrow \operatorname{Hom}_{R}(F, A) \rightarrow \operatorname{Hom}_{R}(F, B) \rightarrow \operatorname{Hom}_{R}(F, C) \rightarrow 0$ of abelian groups is exact for every finitely presented $R$-module $F$. A submodule $A$ of an $R$-module $B$ is called a pure submodule of $B$ if the canonical exact sequence $0 \rightarrow A \rightarrow B \rightarrow B / A \rightarrow 0$ is pure. An $R$-module $M$ is called pure injective if the sequence $0 \rightarrow \operatorname{Hom}_{R}(C, M) \rightarrow \operatorname{Hom}_{R}(B, M) \rightarrow \operatorname{Hom}_{R}(A, M) \rightarrow 0$ is exact for every pure exact sequence $0 \rightarrow A \rightarrow B \rightarrow C \rightarrow 0$ of $R$-modules.

Let $M$ be an $R$-module. $M$ is said to be $\Sigma$-pure injective if for every index set $I$ the direct sum $M^{(I)}$ is pure injective. $M$ is said to be $\Sigma$-self orthogonal if $\operatorname{Ext}_{R}^{1}\left(M, M^{(I)}\right)=0$ for every index set $I$.

The following property of $\Sigma$-pure injective modules will be used in this paper.

Proposition 2.3 ([9], Corollary 1.42, p. 30). Every pure submodule of a $\Sigma$-pure injective module $B$ is a direct summand of $B$.

For unexplained terminology and notation, we refer the reader to $[1,3,8$, $10,13]$.

\section{Properties of Matlis injective modules}

We start with the following definition. 
Definition 3.1. Let $R$ be a ring and $M$ an $R$-module. $M$ is said to be Matlis injective if $\operatorname{Ext}_{R}^{1}(E(R), M)=0$. An $R$-module $N$ is said to be Matlis projective if $\operatorname{Ext}_{R}^{1}(E(R), C)=0$ implies $\operatorname{Ext}_{R}^{1}(N, C)=0$ for any $R$-module $C$. $R$ is said to be a right Matlis ring if $E(R)$ is flat and $p d(E(R)) \leq 1$.

In what follows, we denote by $\mathcal{M I}(\mathcal{M P})$ the class of Matlis injective (projective) $R$-modules. For $\mathcal{C}=\mathcal{M I}, \mathcal{C}$-(pre)envelopes will simply be called Matlis injective (pre)envelopes.

Proposition 3.2. Let $R$ be a ring. Then $\mathcal{M I}$ is closed under extensions, direct products and direct summands; $\mathcal{M I}=\operatorname{Mod}-R$ if and only if $E(R)$ is projective.

Proof. It is easy to see that the assertion holds by definition.

Corollary 3.3. Let $R$ be an integral domain. Then every $R$-module is Matlis injective if and only if $R$ is a field.

Proof. " $\Longleftarrow "$ is trivial.

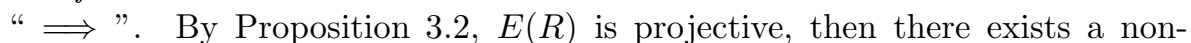
zero homomorphism $f \in \operatorname{Hom}_{R}(E(R), R)$. So $f(E(R))$ is a non-zero divisible submodule of $R$. Let $r$ be any non-zero element from $R$. We choose a non-zero element $x \in f(E(R))$. Since $r x$ is non-zero and $f(E(R))$ is divisible, there is an element $y \in f(E(R))$ with $(r x) y=x$, and so $(r y-1) x=0$. But $R$ is an integral domain, then $r y-1=0$, i.e., $r y=1$. Hence $R$ is a field.

Remark 3.4. Recall that a commutative domain $R$ is called almost perfect provided that $R / I$ is a perfect ring for each ideal $0 \neq I \neq R$. We will show that $\mathcal{M I}$ is not closed under direct sums if $R$ is an almost perfect domain but not a field. If $R$ is an almost perfect domain, then $\mathcal{M I}$ coincides with the class of cotorsion $R$-modules by [10, Theorem 4.4.16, p. 172]. But the class of cotorsion $R$-modules is closed under direct sums if and only if $R$ is a perfect ring by [11, Theorem 19]. Note that $E(R)$ is flat when $R$ is a commutative domain, and so $R$ is a perfect ring if and only if $R$ is a field by Corollary 3.3. Hence $\mathcal{M I}$ is not closed under direct sums when $R$ is an almost perfect domain but not a field. Then we will show that there exist a ring $R$ and an $R$-module $M$ such that $M$ doesn't have a Matlis injective precover. For example, let $R$ be an almost perfect domain but not a field, then there exists a family $\left\{M_{i}\right\}_{i \in I}$ of Matlis injective $R$-modules such that $\bigoplus_{i \in I} M_{i}$ is not Matlis injective. But since $\mathcal{M I}$ is closed under direct summands by Proposition 3.2, it is easy to check that $\bigoplus_{i \in I} M_{i}$ doesn't have a Matlis injective precover.

Lemma 3.5. Let $R$ be a ring. Then every cotorsion $R$-module is Matlis injective if and only if $E(R)$ is flat.

Proof. " $\Longleftarrow "$ is clear.

" $\Longrightarrow$ ". Let $C$ be any cotorsion $R$-module. By hypothesis, we have

$$
\operatorname{Ext}_{R}^{1}(E(R), C)=0 \text {. }
$$

Hence $E(R)$ is flat by the fact that $(\mathscr{F}, \mathscr{C})$ is a cotorsion theory. 
Proposition 3.6. Let $R$ be a ring. Then $\mathcal{M I}=\mathscr{C}$ if and only if $E(R)$ is flat and every Matlis injective $R$-module is cotorsion.

Proof. " $\Longleftarrow "$ holds by assumption and Lemma 3.5.

" $\Longrightarrow$ ". By assumption, we have $M$ is cotorsion if and only if it is Matlis injective. Then the assertion holds by Lemma 3.5.

Proposition 3.7. Let $R$ be a ring. Then the following are equivalent.

(1) Every quotient module of any Matlis injective R-module is Matlis injective.

(2) Every quotient module of any injective R-module is Matlis injective.

(3) The projective dimension of $E(R)$ is at most 1.

Proof. (1) $\Longrightarrow(2)$ is trivial.

$(2) \Longrightarrow(3)$. Let $K$ be any $R$-module. It is enough to show that $\operatorname{Ext}_{R}^{2}(E(R)$, $K)=0$. Let us consider the exact sequence $0 \rightarrow K \rightarrow E(K) \rightarrow E(K) / K \rightarrow 0$. We then have the exact sequence $\operatorname{Ext}_{R}^{1}(E(R), E(K) / K) \rightarrow \operatorname{Ext}_{R}^{2}(E(R), K) \rightarrow$ $\operatorname{Ext}_{R}^{2}(E(R), E(K))=0$. Note that $\operatorname{Ext}_{R}^{1}(E(R), E(K) / K)=0$ by (2), we get $\operatorname{Ext}_{R}^{2}(E(R), K)=0$.

(3) $\Longrightarrow(1)$. Let $M$ be a Matlis injective $R$-module and $N$ a submodule of $M$. Let us consider the exact sequence $0 \rightarrow N \rightarrow M \rightarrow M / N \rightarrow 0$. Applying the functor $\operatorname{Hom}_{R}(E(R),-)$ to the above exact sequence, we get the exact sequence $0=\operatorname{Ext}_{R}^{1}(E(R), M) \rightarrow \operatorname{Ext}_{R}^{1}(E(R), M / N) \rightarrow \operatorname{Ext}_{R}^{2}(E(R), N)$. Note that $\operatorname{Ext}_{R}^{2}(E(R), N)=0$ by (3), so $\operatorname{Ext}_{R}^{1}(E(R), M / N)=0$ and (1) follows.

Remark 3.8. If $E(R)$ is flat, then the condition that every quotient module of any cotorsion $R$-module is Matlis injective is also equivalent to the conditions of Proposition 3.7.

Lemma 3.9. Let $R$ be a ring. Then $(\mathcal{M P}, \mathcal{M I})$ is a cotorsion theory.

Proof. Straightforward.

Theorem 3.10. Let $R$ be a ring. Then the following are equivalent.

(1) $R$ is a right Matlis ring.

(2) Every quotient module of any Matlis injective R-module is Matlis injective and every cotorsion $R$-module is Matlis injective.

(3) Every quotient module of any injective R-module is Matlis injective and every cotorsion $R$-module is Matlis injective.

(4) Every Matlis projective R-module is flat and its projective dimension is at most 1.

Proof. (1) $\Longleftrightarrow(2) \Longleftrightarrow(3)$ hold by Lemma 3.5 and Proposition 3.7.

$(1) \Longrightarrow(4)$. By Lemma 3.9 and [10, Corollary 3.2.4, p. 119], every Matlis projective $R$-module is a direct summand of some $\{E(R), R\}$-filtered $R$-module. Note that every $\{E(R), R\}$-filtered $R$-module is flat and its projective dimension is at most $p d(E(R))$ by (1) and [10, Lemma 3.1.2, p. 113]. So every Matlis projective $R$-module is flat and its projective dimension is at most 1 . 
$(4) \Longrightarrow(1)$. Obviously, $E(R)$ is Matlis projective by definition. So (1) holds by assumption.

Recall that a submodule $N$ of a module $M$ of projective dimension $k$ is said to be a tight submodule if the projective dimension of $M / N$ is at most $k$. We now have the following simple fact:

Proposition 3.11. Let $R$ be a ring. If $p d(E(R)) \leq 1$, then tight submodules of Matlis projective $R$-modules are also Matlis projective.

Proof. Let us consider the exact sequence $0 \rightarrow N \rightarrow M \rightarrow M / N \rightarrow 0$, where $M$ is Matlis projective and $N$ is a tight submodule of $M$. Then $p d(M) \leq 1$ by hypothesis and the proof of Theorem 3.10. For any Matlis injective $R$-module $C$, we have the induced exact sequence

$$
\operatorname{Ext}_{R}^{1}(M, C) \rightarrow \operatorname{Ext}_{R}^{1}(N, C) \rightarrow \operatorname{Ext}_{R}^{2}(M / N, C) .
$$

The two ends vanish, since $M$ is Matlis projective and $p d(M / N) \leq p d(M) \leq 1$. So the middle term is 0 , and hence the assertion holds.

Proposition 3.12. Let $R$ be a ring. Then the following are equivalent.

(1) $C \in \mathcal{M I}$ whenever $0 \rightarrow A \rightarrow B \rightarrow C \rightarrow 0$ is an exact sequence of $R$-modules such that $A, B \in \mathcal{M I}$.

(2) $E(M) / M$ is Matlis injective when $M$ is Matlis injective.

(3) For any $R$-module $M, \operatorname{Ext}_{R}^{1}(E(R), M)=0$ implies $\operatorname{Ext}_{R}^{2}(E(R), M)=$ 0 .

Proof. (1) $\Longrightarrow(2)$ is trivial.

$(2) \Longrightarrow(3)$. Let $M$ be an $R$-module such that $\operatorname{Ext}_{R}^{1}(E(R), M)=0$, i.e., $M$ is Matlis injective. Then $E(M) / M$ is Matlis injective by (2). Applying the functor $\operatorname{Hom}_{R}(E(R),-)$ to the exact sequence $0 \rightarrow M \rightarrow E(M) \rightarrow E(M) / M \rightarrow 0$, we have the exact sequence $0=\operatorname{Ext}_{R}^{1}(E(R), E(M) / M) \rightarrow \operatorname{Ext}_{R}^{2}(E(R), M) \rightarrow$ $\operatorname{Ext}_{R}^{2}(E(R), E(M))=0$. So $\operatorname{Ext}_{R}^{2}(E(R), M)=0$.

$(3) \Longrightarrow(1)$. Let $0 \rightarrow A \rightarrow B \rightarrow C \rightarrow 0$ be an exact sequence of $R$-modules such that $A, B \in \mathcal{M I}$. Applying the functor $\operatorname{Hom}_{R}(E(R),-)$ to the above sequence, we have the exact sequence $0=\operatorname{Ext}_{R}^{1}(E(R), B) \rightarrow \operatorname{Ext}_{R}^{1}(E(R), C) \rightarrow$ $\operatorname{Ext}_{R}^{2}(E(R), A)=0$ by (3). So $\operatorname{Ext}_{R}^{1}(E(R), C)=0$, i.e., $C$ is Matlis injective. Hence (1) holds.

Proposition 3.13. Let $R$ be a commutative Artinian ring. Then $\mathcal{M I}$ is closed under direct sums, pure submodules and direct limits. Moreover, $\mathcal{M I}$ is a definable class, i.e., it is closed under pure submodules, direct products and direct limits.

Proof. By hypothesis, $E(R)$ is finitely presented by [12, Theorem 3.64, p. 90]. Then $\mathcal{M I}$ is closed under direct sums by the isomorphism

$$
\bigoplus \operatorname{Ext}_{R}^{1}\left(F, M_{\alpha}\right) \cong \operatorname{Ext}_{R}^{1}\left(F, \bigoplus M_{\alpha}\right)
$$


for any finitely presented $R$-module $F$ and any family $\left\{M_{\alpha}\right\}$ of $R$-modules. Suppose that $A$ is a pure submodule of a Matlis injective $R$-module $B$. Then we have the exact sequences $0 \longrightarrow \operatorname{Hom}_{R}(E(R), A) \longrightarrow \operatorname{Hom}_{R}(E(R), B) \longrightarrow$ $\operatorname{Hom}_{R}(E(R), B / A) \longrightarrow 0$ and $\operatorname{Hom}_{R}(E(R), B) \longrightarrow \operatorname{Hom}_{R}(E(R), B / A) \longrightarrow$ $\operatorname{Ext}_{R}^{1}(E(R), A) \longrightarrow \operatorname{Ext}_{R}^{1}(E(R), B)=0$. Hence $\operatorname{Ext}_{R}^{1}(E(R), A)=0$, i.e., $A$ is Matlis injective. So $\mathcal{M I}$ is closed under pure submodules. That $\mathcal{M I}$ is closed under direct limits follows from the isomorphism $\operatorname{Ext}_{R}^{1}\left(F, \underline{\lim } M_{i}\right) \cong$

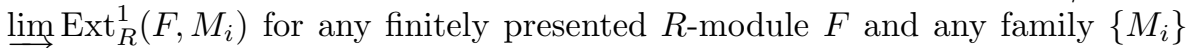
of $R$-modules since $R$ is a commutative Artinian ring. So $\mathcal{M I}$ is definable by Proposition 3.2.

Proposition 3.14. Let $R$ be a commutative Artinian ring and $S \subset R$ be a multiplicative set. If $M$ is a Matlis injective $R$-module, then $S^{-1} M$ is a Matlis injective $S^{-1} R$-module.

Proof. By assumption, $E(R)$ is finitely generated by [12, Theorem 3.64, p. 90] and $R$ is a Noetherian ring. So,

$$
\operatorname{Ext}^{1}{ }_{S^{-1} R}\left(S^{-1} E_{R}(R), S^{-1} M\right) \cong S^{-1} \operatorname{Ext}_{R}{ }_{R}\left(E_{R}(R), M\right)
$$

by $\left[8\right.$, Theorem 3.2 .5 , p. 76]. But $S^{-1} E_{R}(R) \cong E_{S^{-1} R}\left(S^{-1} R\right)$ by [8, Theorem 3.3 .3 , p. 84]. Thus $S^{-1} M$ is a Matlis injective $S^{-1} R$-module when $M$ is a Matlis injective $R$-module.

Proposition 3.15. Let $R$ be a commutative Noetherian ring and $S \subset R$ be a multiplicative set. If $M$ is a Matlis projective $R$-module, then $S^{-1} M$ is a Matlis projective $S^{-1} R$-module.

Proof. Note that $S^{-1} E_{R}(R) \cong E_{S^{-1} R}\left(S^{-1} R\right)$ by [8, Theorem 3.3.3, p. 84] and by hypothesis. Then, every Matlis projective $S^{-1} R$-module is a direct summand of some $\left\{S^{-1} E_{R}(R), S^{-1} R\right\}$-filtered $S^{-1} R$-module by [10, Corollary 3.2 .4$, p. 119]. Since $M$ is a Matlis projective $R$-module, $M$ is a direct summand of some $\{E(R), R\}$-filtered $R$-module by [10, Corollary 3.2.4, p. 119]. Let $N$ be an $\{E(R), R\}$-filtered $R$-module and the chain $\left(N_{\alpha} \mid \alpha \leq \kappa\right)$ be a $\{E(R), R\}$ filtration of $N$. Then $S^{-1} N$ is an $\left\{S^{-1} E_{R}(R), S^{-1} R\right\}$-filtered $S^{-1} R$-module and the chain $\left(S^{-1} N_{\alpha} \mid \alpha \leq \kappa\right)$ is a $\left\{S^{-1} E_{R}(R), S^{-1} R\right\}$-filtration of $S^{-1} N$ by [8, Theorem 1.5.7, p. 33, and Proposition 2.2.4, p. 44] and by definition. So $S^{-1} M$ is a Matlis projective $S^{-1} R$-module and the assertion holds.

\section{The existence of Matlis injective (pre)envelopes}

According to Theorem 2.1, we immediately have the following proposition.

Proposition 4.1. Let $R$ be a ring. Then every $R$-module has a special Matlis injective preenvelope.

The following lemmas are needed to prove the main result of this paper. 
Lemma 4.2. Let $R$ be a ring and $M$ an $R$-module. If $M$ is $\Sigma$-pure injective and $\Sigma$-self orthogonal, then $\operatorname{Add}(M)$ is closed under extensions and direct limits.

Proof. Let $0 \rightarrow A \rightarrow B \rightarrow C \rightarrow 0$ be an exact sequence of $R$-modules such that both $A$ and $C$ are in $\operatorname{Add}(M)$. Without loss of generality, we may assume that both $A$ and $C$ are direct summands of $M^{(I)}$ for an index set $I$. Since $M$ is $\Sigma$-self orthogonal, we have $\operatorname{Ext}_{R}^{1}(C, A)=0$. Then the exact sequence $0 \rightarrow A \rightarrow B \rightarrow C \rightarrow 0$ splits, and so $B \cong A \bigoplus C$. Obviously, $A \bigoplus C \in \operatorname{Add}(M)$. Therefore, $B \in \operatorname{Add}(M)$. So $\operatorname{Add}(M)$ is closed under extensions. We claim that any $R$-module $N$ from $\operatorname{Add}(M)$ is $\Sigma$-pure injective. It is clear that $N$ is pure injective since $M$ is $\Sigma$-pure injective. In addition, $\operatorname{Add}(M)$ is closed under direct sums. Thus $N$ is $\Sigma$-pure injective. Let $\left(\left(M_{i}\right)_{i \in I},\left(f_{j i}\right)\right)$ be a direct system of $R$-modules from $\operatorname{Add}(M)$ where $I$ is a directed set. Then there exists a short exact sequence $0 \rightarrow K \hookrightarrow \bigoplus_{i \in I} M_{i} \rightarrow \lim _{i} \rightarrow 0$ with $K$ a pure submodule of $\bigoplus_{i \in I} M_{i}$. But $\bigoplus_{i \in I} M_{i}$ is $\Sigma$-pure injective, then the exact

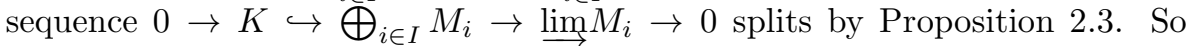
$\underset{\lim }{\longrightarrow} M_{i}$ is isomorphic to a direct summand of $\bigoplus_{i \in I} M_{i}$, i.e., $\underset{\lim }{\longrightarrow} M_{i} \in \operatorname{Add}(M)$. Hence $\operatorname{Add}(M)$ is closed under direct limits.

Lemma 4.3. Let $R$ be a ring and $M$ an $R$-module. Assume that $M$ is $\Sigma$-pure injective and $\Sigma$-self orthogonal. Then every $R$-module $N$ has an $M^{\perp_{1}}$-envelope.

Proof. Obviously, $M^{\perp_{1}}=(\operatorname{Add}(M))^{\perp_{1}}$. Thus it is equivalent to show that every $R$-module $N$ has an $(\operatorname{Add}(M))^{\perp_{1}}$-envelope. By Theorem $2.1, N$ has a special $(\operatorname{Add}(M))^{\perp_{1}}$-preenvelope $f$ with Coker $f$ is $\{M\}$-filtered. Note that every $\{M\}$-filtered $R$-module is in $\operatorname{Add}(M)$ by Lemma 4.2 and transfinite induction. So $N$ has an $(\operatorname{Add}(M))^{\perp_{1}}$-envelope by Lemma 4.2 and Theorem 2.2.

We are now in a position to prove the following

Theorem 4.4. Let $R$ be a right Noetherian ring and $\mathcal{F}$ a class of injective $R$ modules. Then every $R$-module $M$ has an $\mathcal{F}^{\perp_{1}}$-envelope; in particular, every $R$-module $M$ has a Matlis injective envelope.

Proof. If $R$ is right Noetherian, then every injective $R$-module is the direct sum of indecomposable injective $R$-modules. Each such module is the injective envelope of a cyclic $R$-module. Hence, we can find a representative set of such modules. So there is a family $\left\{E_{i}\right\}_{i \in I}$ of indecomposable injective $R$-modules such that every injective $R$-module is the direct sum of copies of $E_{i}$.

Let $\mathcal{S}=\left\{E_{i} \mid E_{i}\right.$ is isomorphic to a direct summand of an element of $\left.\mathcal{F}\right\}$. It is easy to see that $\left(\bigoplus_{E_{i} \in \mathcal{S}} E_{i}\right)^{\perp_{1}}=\mathcal{F}^{\perp_{1}}$. Note that $\bigoplus_{E_{i} \in \mathcal{S}} E_{i}$ is $\Sigma$-pure injective and $\Sigma$-self orthogonal by the fact that the class of right injective $R$-modules is closed under direct sums when $R$ is right Noetherian. So the assertion holds by Lemma 4.3 .

We end this paper with the following remark. 
Remark 4.5. If $R$ is a commutative Artinian ring, then every $R$-module has a Matlis injective cover by Proposition 3.13 and [2, Corollary 2.6 and Proposition $4.3(3)]$.

Acknowledgements. The author would like to thank the referees for the very helpful comments and suggestions.

\section{References}

[1] F. W. Anderson and K. R. Fuller, Rings and Categories of Modules, Grad. Texts in Math. 13, Springer-Verlag, New York 1974.

[2] S. Bazzoni, When are definable classes tilting and cotilting classes?, J. Algebra 320 (2008), no. $12,4281-4299$.

[3] H. Cartan and S. Eilenberg, Homological Algebra, Princeton Math. Ser., 1956.

[4] P. C. Eklof and S. Shelah, On Whitehead modules, J. Algebra 142 (1991), no. 2, 492-510.

[5] P. C. Eklof and J. Trlifaj, How to make Ext vanish, Bull. Lond. Math. Soc. 23 (2001), no. $1,41-51$.

[6] E. E. Enochs, Injective and flat covers, envelopes and resolvents, Israel J. Math. 39 (1981), no. 3, 189-209.

[7] E. E. Enochs and O. M. G. Jenda, Copure injective modules, Quaest. Math. 14 (1991), no. 4, 401-409.

[8] — Relative Homological Algebra, de Gruyter Expos. Math. 30, de Gruyter, Berlin 2000.

[9] A. Facchini, Module Theory: Endomorphism Rings and Direct Sum Decompositions in Some Classes of Modules, Progress in Math. vol. 167, Birkhäuser, Basel, 1998.

[10] R. Göbel and J. Trlifaj, Approximations and Endomorphism Algebras of Modules, de Gruyter Expos. Math. 41, de Gruyter, Berlin 2006.

[11] P. A. Guil Asensio and I. Herzog, Sigma-cotorsion rings, Adv. Math. 191 (2005), no. 1, $11-28$.

[12] T. Y. Lam, Lectures on Modules and Rings, Grad. Texts in Math. 189, Springer, New York 1999.

[13] J. J. Rotman, An Introduction to Homological Algebra, Pure Appl. Math. 85, Academic Press, New York 1979.

FACUlty OF SCIEnCE

China Pharmaceutical University

NANJING 211198, P. R. China

E-mail address: hyyan07@126.com 